\title{
Aortic-ventricular tunnel in a neonate: diagnosis and management based on cross sectional and colour Doppler ultrasonography
}

\author{
N Sreeram, R Franks, K Walsh
}

\begin{abstract}
A five day old symptom free neonate was referred for assessment of a to and fro murmur associated with large volume pulses. Cross sectional echocardiography and colour flow mapping confirmed the diagnosis of an aortic-ventricular tunnel with forward flow into the aorta and regurgitant flow into the ventricle through both the tunnel and the dilated aortic valve ring. Surgical correction by patch closure of the aortic end of the tunnel was successfully undertaken two weeks later without any additional investigations. Postoperative echocardiography and colour flow imaging showed no aortic regurgitation and normal left ventricular dimensions and function.
\end{abstract}

An aortic-ventricular tunnel is a rare congenital malformation that presents with clinical signs of aortic regurgitation and may produce cardiac failure in infancy. There are a few reports of successful diagnosis by cross sectional and Doppler ultrasound and the diagnosis has been based mainly on the demonstration of the tunnel by aortic root angiography. ${ }^{1-3}$ It may, however, be difficult to show the intracardiac portion of the tunnel, particularly when there is gross distortion of the left ventricular outflow tract. In addition, angiography is not without risks in the sick neonate. We describe a neonate in whom the diagnosis of an aortic-ventricular tunnel was
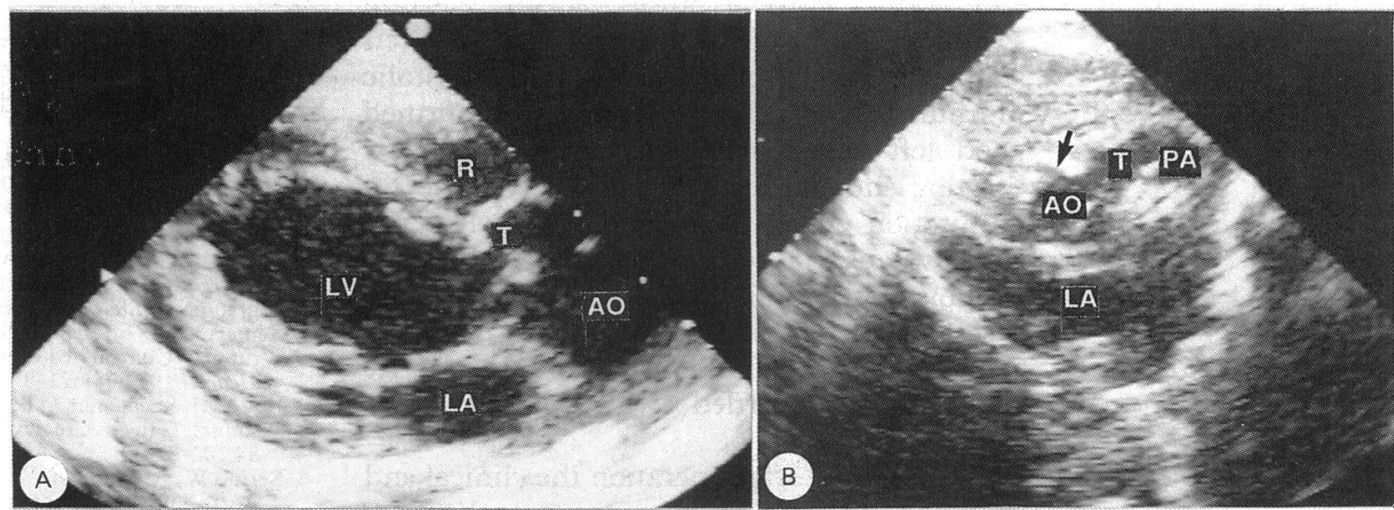

Figure 1(A) Cross sectional echocardiogram in the parasternal long axis view showing the entire extent of the tunnel between the aorta and left ventricle. AO, aorta; $L A$, left atrium; $L V$, left ventricle; $R$, right ventricular outflow tract; $T$, tunnel. (B) Cross sectional echocardiogram in the parasternal short axis view at the level of the aortic root showing the relation of the tunnel to the right coronary artery (arrow) and coronary sinus. $A O$, aorta; $L A$, left atrium;

$P A$, pulmonary artery; $T$, tunnel. confirmed by cross sectional echocardiography with Doppler colour flow imaging, and successful surgical repair was undertaken on the basis of these investigations alone.

\section{Case report}

A five day old female infant weighing $2.7 \mathrm{~kg}$ was referred for the assessment of a to and fro murmur that had been noted at routine examination after an uncomplicated vaginal delivery. At examination she was symptom free but had bounding pulses associated with an ejection systolic and early diastolic murmur grade III that was best heard in the third intercostal space at the left sternal edge. The chest $x$ ray showed cardiomegaly (cardiothoracic ratio 0.68 ) with a widened mediastinal shadow; the electrocardiogram showed left ventricular hypertrophy with non-specific changes in the ST segment and T wave.

\section{ECHOCARDIOGRAPHY}

Cross sectional echocardiography showed a dilated left ventricle with concentric hypertrophy but good contractility. In the parasternal long axis view a tunnel could be seen arising from the region of the right aortic sinus and passing between the aortic valve and the outlet portion of the ventricular septum to terminate in the left ventricle (fig 1A). Parasternal short axis scans at the level of the aortic root showed the relation of the tunnel to the right aortic sinus and coronary artery (fig 1B). The aortic valve had three cusps of equal
Heart Clinic, Royal Liverpool Children's N Sreeram R Franks

Correspondence to

Dr N Sreeram, Heart Clinic, Royal Liverpool Children's Hospital, Alder Hey, Eaton
Road, Liverpool L12 2AP. 


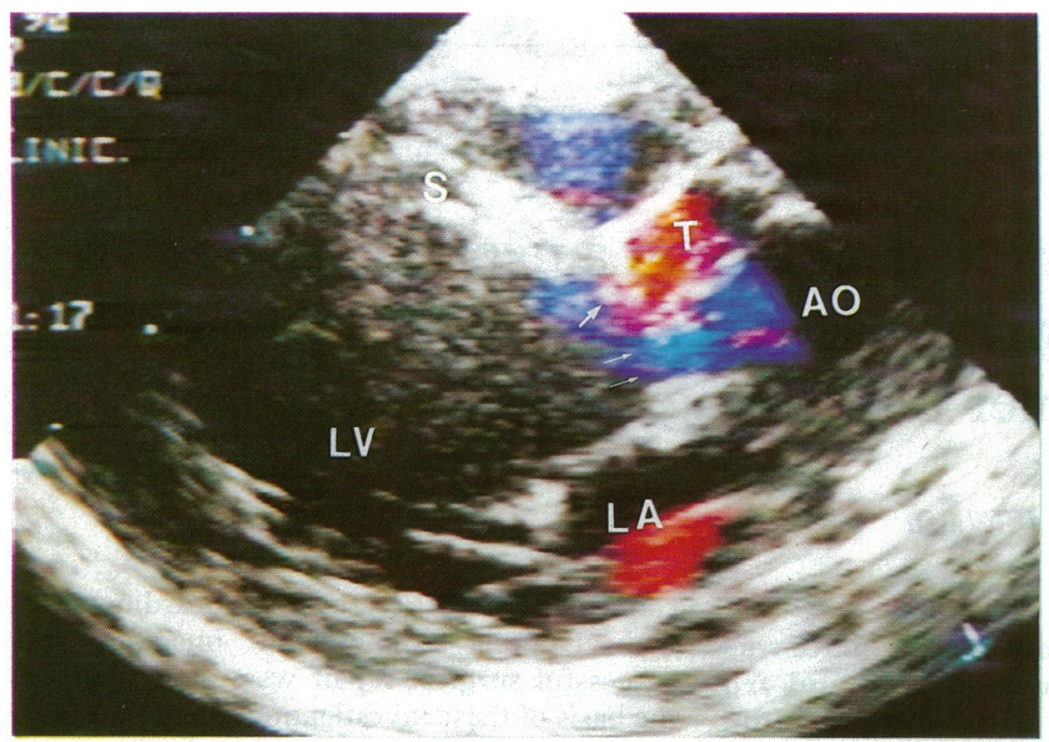

Figure 2 Doppler colour flow imaging in the parasternal long axis view. Forward flow through both the aortic valve (double arrows) and the tunnel (single arrow) are shown in this systolic frame. Regurgitation through both the tunnel and the dilated valve ring was seen in diastole. $A O$, aorta; $L A$, left atrium; $L V$, left ventricle; $S$, interventricular septum; T, tunnel. the chest $x$ ray. All treatment for heart failure had been stopped.

\section{Discussion}

A tunnel between the aorta and left ventricle is a rare malformation in which a communication between the aorta and the left ventricle bypasses the aortic valve. The lesion typically presents with a to and fro murmur and has to be considered whenever isolated aortic regurgitation is suspected in infancy. The natural course depends upon the degree of aortic regurgitation. In general, there is progressive heart failure and the patient dies if surgical intervention is not undertaken, ${ }^{4}$ though the occasional patient has survived to adult life apparently free of symptoms. While the immediate results of surgery have been good, long term follow up of patients treated surgically seems to show that a considerable proportion of patients have persistent aortic valve regurgitation after closure of the tunnel and require further surgery. ${ }^{5}$ Most patients with persistent aortic valve regurgitation, however, are older at operation and by then there is considerable dilatation of the aortic root and distortion of the aortic valve apparatus caused both by left ventricular dilatation and longstanding high velocity turbulent flow through the tunnel. In contrast, in the only report on long term follow up after operation in infancy, no aortic regurgitation was seen five years after operation, despite persistence of the blind ended intramural portion of the tunnel. ${ }^{6}$ Early repair of the lesion is therefore indicated, even when there are no symptoms. The onset of cardiac failure two weeks after initial diagnosis in this previously well infant shows the unpredictable clinical course.

Colour Doppler flow mapping was useful in identifying and distinguishing regurgitation through both the tunnel and the aortic valve, which angiography often fails to do. The combination of colour Doppler flow mapping and high resolution cross sectional imaging should reliably identify most coexisting defects. This technique is also useful for serial non-invasive follow up assessment of left ventricular and aortic valve function. Such studies of a larger series of patients operated on in infancy are needed to determine whether early operation reduces the frequency of aortic valve regurgitation and resultant morbidity.

1 Fripp RR, Werner JC, Whitman V, Nordenberg A, Waldhausen JA. Pulsed Doppler and two-dimensional echocardiusen fic findings in aortico-left ventricular tunnel. Am Coll Cardiol 1984;4:1012-4.

2 Hucin B, Samanek M. Correction of aortico-left ventricular tunnel during the Correction of aortico-left ventricular tunnel durin

3 first day of life. Ann Thorac Surg 1989 . Aorto-left ventricular tunnel. Clinical features and surgical management. $B r$ tunnel. Clinical features

4 Levy MJ, Schachner A, Blieden LC. Aortico-left ventricular tunnel: collective review. J Thorac Cardiovasc Surg 1982; tunnel: collec

84:102-9. Serino W, Andrade JL, Ross D, de Leval M, Somerville J.
Aorto-left ventricular communication after closure. Late postoperative problems. Br Heart $J$ 1983;49:501-6.

6 Bjork V, Eklof O, Wallgren G, Zetterqvist P. Successful surgical treatment of an aortico-left ventricular tunnel in a four-month old infant. $J$ Thorac Cardiovasc Surg 1979 78:35-8. echocardiographic findings The electrocardiogram showed regression of the left ventricular voltages associated with a decrease in the cardiothoracic ratio $(0 \cdot 6)$ on 